\title{
Why do we have a reconnected right pulmonary vein after contact force-guided ablation? Intercaval bundle bypassing a durable circumferential lesion
}

\author{
Satoshi Aita ${ }^{1}$, Hiro Yamasaki ${ }^{1}$, Akihiko Nogami ${ }^{1}$, and Masaki Ieda ${ }^{1}$ \\ ${ }^{1}$ University of Tsukuba
}

May 29, 2020

\section{EP Rounds}

Why do we have a reconnected right pulmonary vein after contact force-guided ablation? Intercaval bundle bypassing a durable circumferential lesion

Satoshi Aita, $\mathrm{MD}^{1}$, Hiro Yamasaki, $\mathrm{MD}^{2}, \mathrm{PhD}$, Akihiko Nogami, $\mathrm{MD}^{2}, \mathrm{PhD}$, Masaki Ieda, MD, $\mathrm{PhD}^{2}$

${ }^{1}$ Division of Cardiovascular Medicine, Tsukuba Medical Center Hospital, Tsukuba, Japan

${ }^{2}$ Department of Cardiology, Faculty of Medicine, University of Tsukuba, Tsukuba, Japan

\section{Address for correspondence :}

Hiro Yamasaki, MD

Department of Cardiology, Faculty of Medicine, University of Tsukuba

1-1-1 Tennodai, Tsukuba, Ibaraki 305-8575, Japan

Tel: $+81-29-853-3141$

Fax: +81-29-853-3143

E-mail:hyamasaki@md.tsukuba.ac.jp

Conflict of interest: None

Funding: None

\section{Case Presentation}

A 67-year-old woman with persistent atrial fibrillation (AF) underwent pulmonary vein isolation using a contact force-sensing catheter (SmartTouch SF, Biosense Webster, CA). We performed an ablation index (AI)-guided circumferential energy application (AI of $>400$ at the posterior wall roof and $>450$ at the anterior wall) with a target lesion distance of $4 \mathrm{~mm}$. However, the right pulmonary vein (PV) potential remained after the circumferential lesion. The time difference between the P-wave onset to the right superior PV (RSPV) remained constant. From the activation sequence of a circular mapping catheter placed in the RSPV, the earliest activation site was the anterior carina. Additional energy applications were delivered just inside the initial anterior ablation line (Figure 1A). The right superior and inferior PVs were individually isolated, but they did not require linear carina ablation. After 5 months, the patient was readmitted to the hospital for recurrent $\mathrm{AF}$. 
Repeat ablation was performed using a three-dimensional mapping system (CARTO3, Biosense Webster) and reconnection of the RPV was detected. An activation sequence of a circular mapping catheter placed in the RSPV demonstrated the earliest activation site to be the anterior carina. The time difference between $\mathrm{P}$ wave onset and RSPV potential was $48 \mathrm{~ms}$, and a conduction delay was not apparent despite the previous ablation. Detailed mapping of the left atrium (LA) was performed using a 1-mm multi-electrode mapping catheter (PentaRay ${ }^{\circledR}$, Biosense Webster) during sinus rhythm. Activation mapping within a circumferential line demonstrated that the earliest activation site was the anterior carina. This suggested a conduction breakthrough over the anterior line where the myocardium was thick (Figure 1B ). However, a voltage map demonstrated a contiguous circumferential lesion without an apparent gap (Figure 1C ). Where is the gap and where should we ablate?

\section{Commentary}

A previous study has demonstrated that during sinus rhythm, conduction from the right atrium (RA) to the LA occurs via three distinct sites-Bachmann's bundle, the rim of the fossa ovalis, and the coronary sinus. ${ }^{1}$ However, anatomical and electrophysiological studies have reported a fourth interatrial conduction over an intercaval bundle that connects the RA and the carina of the right $\mathrm{PVs}^{2,3}$ In the era of contact force-guided ablation, a contiguous and durable lesion is expected. ${ }^{4}$ Nevertheless, the intercaval bundle that connects the RA and the carina of the right PV bypasses the durable circumferential lesion. Therefore, when performing repeat ablation after contact force-guided ablation that requires additional ablation in the carina, AF recurrence due to reconnection over the intercaval conduction should also be considered a possible mechanism of recurrence.

In this case, the earliest LA breakthrough was found at the insertion site of Buchmann's bundle. The activation timing of the anterior carina of the right PV was slightly delayed compared to that of Bachmann's bundle insertion site. Hence, we suspected a residual conduction over a previous circumferential region. However, several energy applications delivered on the circumferential line were unsuccessfully. Therefore, we considered the possibility of an electrical connection over the intercaval bundle. Initially, an activation map was created in the LA during pacing from the RSPV (Figure 2 ). The earliest activation in the LA was found at the LA breakthrough site of Bachmann's bundle during sinus rhythm, and a collision activation pattern was observed along the circumferential ablation line. This finding suggested that pacing from the RSPV was blocked at the anterior line, but it was conducted to the RA via the intercaval bundle and then propagated over Buchmann's bundle. We, therefore, created a further activation map for the RA. The earliest activation site was at the posterior wall of the RA, anatomically opposite to the right PV carina. Initial energy applications at the earliest RA activation site prolonged the RSPV potential, and the second energy application achieved ipsilateral right PV isolation (Figure 3 ). No further energy applications were required in the LA.

Contiguous and optimized radiofrequency energy applications improve lesion durability. Nevertheless, in the presence of an RA-RPV intercaval bundle, additional energy application within a circumferential line is required to achieve PV isolation. In the presence of reconduction over the intercaval bundle, activation mapping or activation sequencing of a circular mapping catheter demonstrates the earliest activation site at the anterior carina. This activation might be misinterpreted as residual conduction over the anterior line where the thick myocardium exits. A recent study has demonstrated the importance of detailed activation mapping before initial ablation. ${ }^{5}$ Patients with two early activation sites - Bachmann's bundle and the anterior carina - frequently required carina ablation, suggesting an epicardial connection. ${ }^{5}$ However, detailed activation mapping may not be helpful for identifying the earliest activation site of the LA during repeated procedures. Precise annotation is limited where energy applications were performed previously. Even a slight conduction delay after a previous ablation may unmask an interatrial connection over the intercaval bundle. When performing a repeated procedure after AI-guided ablation requiring RPV carina ablation, it is important to consider possible reconduction over the intercaval conduction. Activation mapping during pacing from the RSPV might help distinguish the reconnection site. Targeting the RA insertion site of the intercaval bundle avoids the risk of PV stenosis and can, thus, be considered an alternative target. 


\section{Figure Legends}

Figure 1 A. Ablation points during the first procedure.B. Activation mapping during sinus rhythm demonstrated reconnection of the right superior pulmonary vein. The earliest activation site within a previous circumferential was found in the anterior carina area where the pulmonary vein was isolated during the initial procedure. The earliest activation in the left atrium was found at the insertion site of Bachmann's bundle. C. Voltage mapping during repeated ablation without an apparent gap along the ablation line. RIPV = right inferior pulmonary vein, RSPV = right superior pulmonary vein

Figure 2. Activation mapping during pacing from the right superior pulmonary vein. The earliest activation site was at the posterior wall of the right atrium, opposite the right pulmonary vein carina. The earliest activation in the LA was similar to that during sinus rhythm, suggesting conduction over Bachmann's bundle.

$\mathrm{LA}=$ left atrium, $\mathrm{RA}=$ right atrium

Figure 3 A Fluoroscopic views of the successful isolation site.B. Intracardiac electrocardiogram of the successful ablation site.

$\mathrm{ABL}=$ ablation; $\mathrm{CS}=$ coronary sinus; $\mathrm{HRA}=$ high right atrium; $\mathrm{LAO}=$ left anterior oblique; $\mathrm{RAO}=$ right anterior oblique; RSPV = right superior pulmonary vein

\section{References}

1. Lemery R, Soucie L, Martin B, Tang AS, Green M, Healey J. Human study of biatrial electrical coupling: determinants of endocardial septal activation and conduction over interatrial connections. Circulation 2004;110:2083-2089.

2. Ho SY, Cabrera JA, Sanchez-Quintana D. Left atrial anatomy revisited. Circ Arrhythm Electrophysiol 2012;5:220-228.

3. Patel PJ, D'Souza B, Saha P, Chik WW, Riley MP, Garcia FC. Electroanatomic mapping of the intercaval bundle in atrial fibrillation. Circ Arrhythm Electrophysiol 2014;7:1262-1267.

4. Phlips T, Taghji P, El Haddad M, Wolf M, Knecht S, Vandekerckhove Y, Tavernier R, Duytschaever M. Improving procedural and one-year outcome after contact force-guided pulmonary vein isolation: the role of interlesion distance, ablation index, and contact force variability in the 'CLOSE'-protocol. Europace 2018;20:f419-f427.

5. Yoshida K, Baba M, Shinoda Y, Harunari T, Tsumagari Y, Koda N, Hayashi K, Yaguchi T, Watabe H, Hasebe H, Aonuma K, Takeyasu N, Nogami A, Ieda M. Epicardial connection between the right-sided pulmonary venous carina and the right atrium in patients with atrial fibrillation: A possible mechanism for preclusion of pulmonary vein isolation without carina ablation. Heart Rhythm 2019;16:671-678.

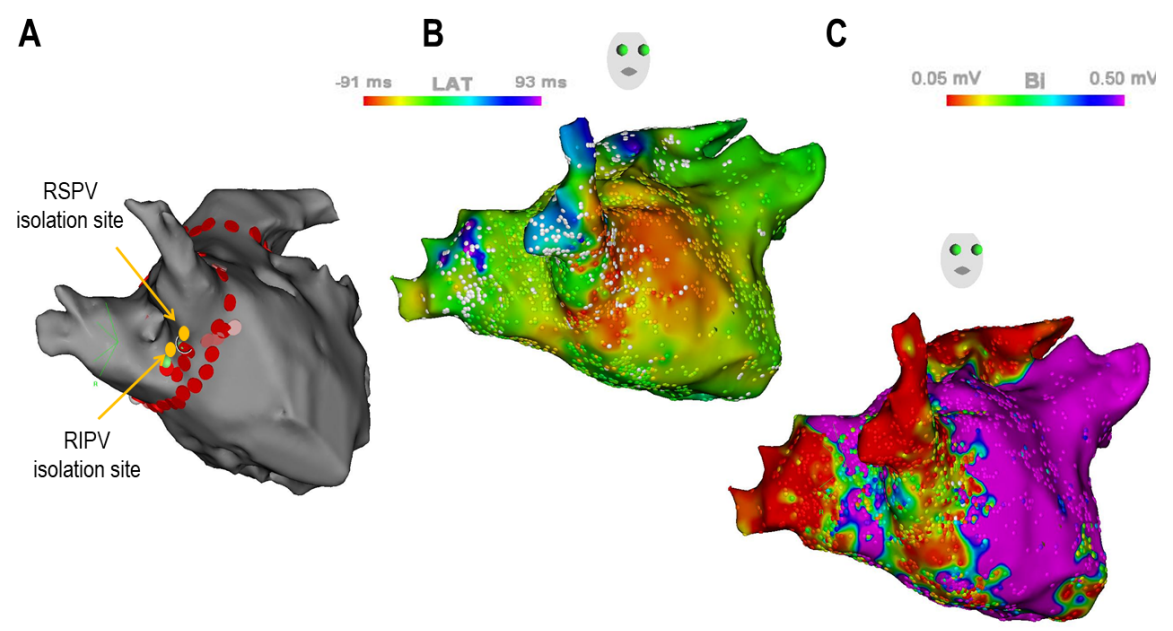



